April 29, 2018

LBNL-42429

UCB-PTH-98/53

hep-ph/9810468

\title{
Tau Appearance In Atmospheric Neutrino Interactions
}

\author{
Lawrence J. Hall and Hitoshi Murayama \\ Department of Physics \\ University of California, Berkeley, California 94720 \\ Theoretical Physics Group \\ Ernest Orlando Lawrence Berkeley National Laboratory \\ University of California, Berkeley, California 94720
}

\begin{abstract}
If the correct interpretation of the Super-Kamiokande atmospheric neutrino data is $\nu_{\mu} \rightarrow \nu_{\tau}$ oscillation, the contained data sample should already have more than $10 \tau$ appearance events. We study the challenging task of detecting the $\tau$, focussing on the decay chain $\tau^{ \pm} \rightarrow$ $\rho^{ \pm} \rightarrow \pi^{ \pm} \pi^{0}$ in events with quasi-elastic $\tau$ production. The background level, which is currently quite uncertain because of a lack of relevant neutral current data, can be measured by the near detector in the K2K experiment. Our estimates of the background suggest that it may be possible to detect $\tau$ appearance in Super-Kamiokande with 5-10 years of running.
\end{abstract}

\footnotetext{
${ }^{*}$ This work was supported in part by the U.S. Department of Energy under Contracts DE-AC03-76SF00098, in part by the National Science Foundation under grant PHY-9514797. HM was also supported by Alfred P. Sloan Foundation.
} 


\section{Introduction}

Recent data from the Super-Kamiokande (SK) detector [1] has provided confirmation of the atmospheric neutrino anomaly, previously reported by Kamiokande [2] and IMB [3] collaborations. The Soudan-II [4] and MACRO [5] Collaborations have also reported anomalies which support the SuperKamiokande observations. Apparently about $40 \%$ of the $\nu_{\mu}$ produced in cosmic ray showers in the atmosphere do not reach the underground detectors. While calculations of the $\nu_{\mu}$ flux contains uncertainties, these calculations can be checked by comparing with the observed rate of muon and $\nu_{e}$ induced events. Neutrino oscillations provide a plausible depletion mechanism which has been strengthened by recent Super-Kamiokande data showing that the amount of $\nu_{\mu}$ depletion depends on the azimuthal angle.

In this letter, we argue that, if the oscillation is to $\nu_{\tau}$, then the SuperKamiokande detector could detect charged current $\tau$ appearance events via the chain $\nu_{\tau} \rightarrow \tau^{-} \rightarrow \rho^{-} \rightarrow \pi^{-} \pi^{0}$. The signal event rate is small because only a small fraction of $\nu_{\tau}$ have sufficient energy to produce $\tau$. Furthermore, there is considerable uncertainty in the background from $\rho$ production via other neutrino induced processes. However, we estimate the signal to background ratio to be above unity, and the signal has a strong up-down asymmetry. A multi-year search at Super-Kamiokande with an efficiency of $50 \%$ could lead to a $5 \sigma$ signal.

The atmospheric $\nu_{\mu}$ could oscillate into $\nu_{e}, \nu_{\tau}$ or $\nu_{s}$, an $\mathrm{SU}(2)$ singlet neutral fermion. Fits to the atmospheric data, in models with three generations and a single $\Delta m^{2}$ relevant to the atmospheric oscillation, show that at most about a third of the oscillations can be to $\nu_{e}$, so that at least two thirds are to $\nu_{\tau}$ [6]. For values of $\Delta m^{2}$ above $2 \times 10^{-3} \mathrm{eV}^{2}$, the CHOOZ experiment constrains the $\nu_{e}$ fraction to be even smaller [7]. Within the minimal scenario, the oscillations are dominantly to $\nu_{\tau}$. While oscillations to $\nu_{s}$ remain a possibility, it is theoretically less favored.

How can the preferred $\nu_{\mu} \rightarrow \nu_{\tau}$ interpretation be verified? A long baseline experiment with low neutrino beam energy (e.g., KEK to SK) would see $\nu_{\mu}$

\footnotetext{
$\dagger$ It requires the addition of an exotic fermion, neutral under the known gauge interactions. Many theories contain such fermions; however, because of their neutrality, they typically get very large masses, thereby inducing small masses for $\nu_{e, \mu, \tau}$ by the see-saw mechanism. On the other hand, for $\nu_{\mu}$ to oscillate into $\nu_{s}$, the mass of $\nu_{s}$ must certainly be less than $250 \mathrm{keV}$. Understanding such a light mass for a singlet state is possible, but requires more elaborate theoretical structures.
} 
disappearance but not $\tau$ appearance. For higher energy neutrino beams (e.g., MINOS and ICARUS) the more direct signal of $\tau$ appearance is possible. In this letter we argue that a $\tau$ appearance signal, with a large up-down asymmetry, may be possible at Super-Kamiokande. We caution that the event rates are low, and that a significant $\rho$ detection efficiency is necessary. It may be possible to see this signal even if $\Delta m^{2} \leq 10^{-3} \mathrm{eV}^{2}$, where the planned accelerator based experiments become more difficult.

\section{$2 \tau$ Appearance}

In this section, we give numerical results for $\tau$-appearance cross sections, from both quasi-elastic (QE) processes

$$
\begin{aligned}
& \nu_{\tau} n \rightarrow \tau^{-} p, \\
& \bar{\nu}_{\tau} p \rightarrow \tau^{+} n,
\end{aligned}
$$

and deep inelastic scattering (DIS) processes

$$
\begin{aligned}
& \nu_{\tau} p \rightarrow \tau^{-} X, \\
& \nu_{\tau} n \rightarrow \tau^{-} X, \\
& \bar{\nu}_{\tau} p \rightarrow \tau^{+} X, \\
& \bar{\nu}_{\tau} n \rightarrow \tau^{+} X .
\end{aligned}
$$

We have not calculated the additional contributions from resonance production. Therefore, the sum of the QE and DIS contributions gives a conservative estimate of the total $\tau$ production cross section.

The QE cross sectionsare evaluated using the formula in Ref. [8], with the second-class currents dropped, PCAC and dipole form factors assumed, and taking $m_{A} \simeq 1.0 \mathrm{GeV}$ as suggested by experiments [9]. The results are shown in Fig. 1 together with the corresponding quasi-elastic cross sections for muon (anti-)neutrino induced events. We quote cross sections for $\tau$ production at $E_{\nu}=5 \mathrm{GeV}:$

$$
\begin{aligned}
\sigma_{Q E}\left(\nu_{\tau} n \rightarrow \tau^{-} p\right) & =3.5 \mathrm{fb} \\
\sigma_{Q E}\left(\bar{\nu}_{\tau} p \rightarrow \tau^{+} n\right) & =2.0 \mathrm{fb} .
\end{aligned}
$$

The DIS cross sections are evaluated using the MRS-A parton luminosities, specifically extended to the low- $Q^{2}$ regime [10]. The parton-level cross 


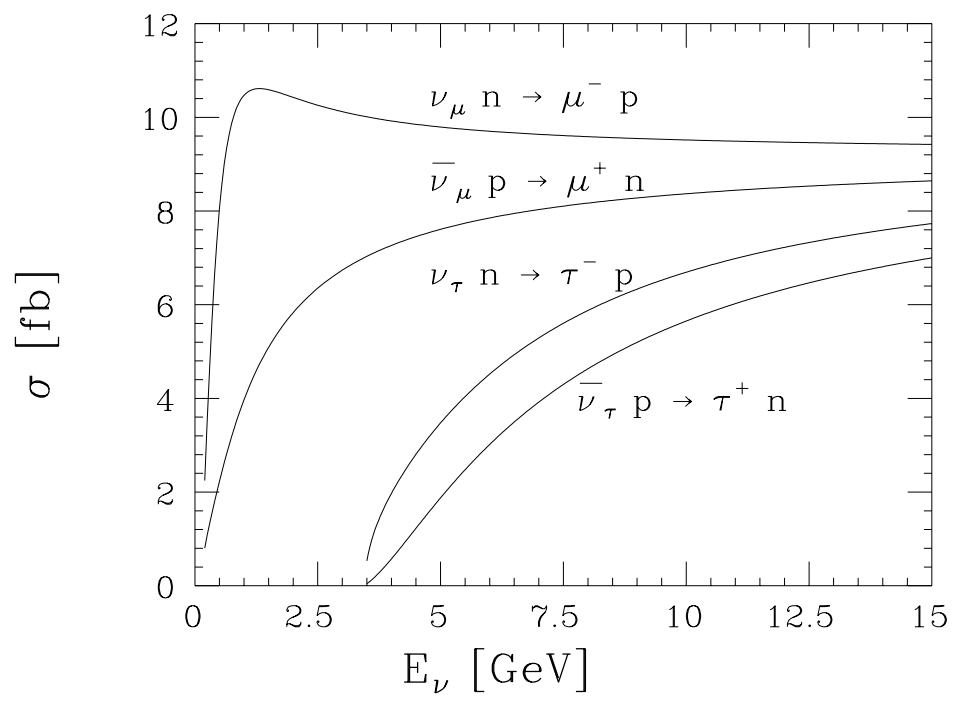

Figure 1: The cross sections of $\tau$ production from $\nu_{\tau}$ charged current reactions, for quasi-elastic (QE) events as described in the text. The $\mu$ production cross sections are shown for comparison.

sections, however, are the leading-order calculations [11]. Cross sections for all four processes, (3,4, 1, 3, 6) are shown in Fig. 2. .

The QE cross sections are combined with calculations of the atmospheric neutrino flux [12 for a 414 day exposure at SuperKamiokande (21.1 kton), assuming no nuclear effects. The total number of electron events calculated is 106.7 for the multi-GeV sample, $p_{e}>1.33 \mathrm{GeV}$. According to the $\mathrm{MC}$ calculations based on the same atmospheric neutrino flux 12 shown in the 2nd paper in [1], the Super-Kamiokande collaboration expect 182.7 e-like events, with $38.3 \%$ from the QE process. Therefore, we overestimate the QE event rate, due to our lack of knowledge of the detection efficiency and the nuclear shadowing effect, and we should multiply our calculated results by a reduction factor of 0.66 .

To confirm this reduction factor, we further compared the multi-GeV $\mu-$

\footnotetext{
${ }^{\ddagger}$ In all of our discussions, we took the $\tau$ threshold from nucleons at rest. In reality, however, the Fermi motion of nucleons inside oxygen nuclei lowers the threshold and hence the expected signal rate is higher. We thank G. Shapiro on this point. A precise estimate of this effect, however, requires a nuclear model and is beyond the scope of this paper. It is worth emphasizing though that we estimate the signal rate conservatively only using QE process with fixed production threshold.
} 


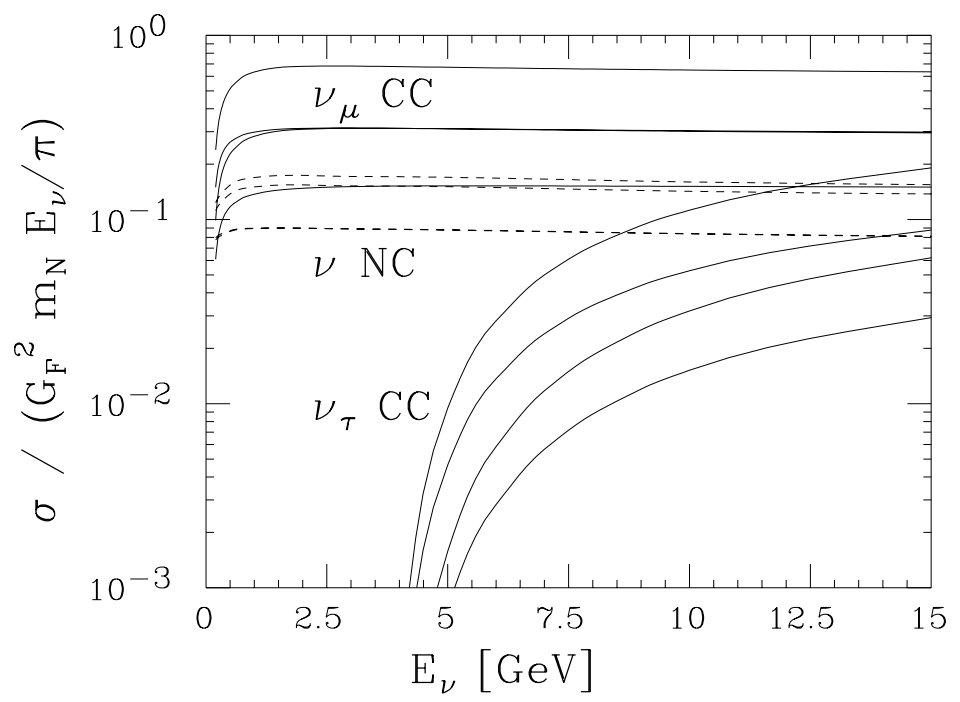

Figure 2: The cross sections of $\tau$ production from $\nu_{\tau}$ charged current reactions for deep-inelastic (DIS) events, normalized by $G_{F}^{2} m_{N} E_{\nu} / \pi$. The rising solid curves $\left(\nu_{\tau} \mathrm{CC}\right)$ are for $\bar{\nu}_{\tau} n, \nu_{\tau} p, \bar{\nu}_{\tau} p$, and $\nu_{\tau} n$ from below. The neutral current $(\nu \mathrm{NC})$ processes are given in dashed lines, for $\bar{\nu} p(n), \nu p, \nu n$ from below; the first two are accidentally close and are not distinguishable in the plot. The $\nu_{\mu}$ CC production cross sections are shown for comparison, for $\nu_{\mu} n, \nu_{\mu}\left(\bar{\nu}_{\mu}\right) p$, and $\bar{\nu}_{\mu} n$ from above, where the middle two are accidentally close and are not distinguishable in the plot. 
like events to our calculations. This is slightly more complicated because there are both fully contained (FC) and partially contained (PC) events. We find that there should be 250.0 QE events. Their MC shows 229.0 FC events, with a $54.1 \%$ QE fraction, and 287.7 PC events with 17.9\% QE: in total, 175.5 QE events. The same reduction factor of 0.66 explains the descrepancy (actually about 0.7), and hence we adopt this factor to scale between our calculated rates and those from the Super-Kamiokande Monte Carlo.

The Super-Kamiokande Collaboration observed $218 e$-like multi-GeV events in 414 days. This is larger than the 182.7 events expected from their Monte Carlo calculation by a factor of 1.19 . We assume that this has nothing to do with oscillations, but is due to the uncertainties in the flux calculation. We therefore assume that all unoscillated fluxes, produced in the cosmic ray showers, are a factor 1.19 larger than calculated in [12], which increases our reduction factor from 0.66 to 0.77 .

We studied the momentum distribution of $e$ and $\mu$ events after rescaling the normalization as discussed, for a 414 day exposure at SuperKamiokande (21.1 kton). This distribution for $\mu$ events is shown in Fig. 3(a). Compared to the reported data, the fraction of QE events in the one-ring event samples becomes smaller for higher momentum as expected on physical grounds. The other events correspond to the production of a few additional soft particles below the detection threshold. We will come back to this issue later. 13

Including $\nu_{\mu}-\nu_{\tau}$ oscillations for the "test point" used by Super-Kamiokande

$$
\Delta m^{2}=0.005 \mathrm{eV}^{2}, \sin ^{2} \theta=1,
$$

we obtain the dashed histogram in Fig. 3(a) for the muon momentum distribution. Correspondingly, we find $\tau$-appearance from the QE reaction as shown in Fig. 3(b). The event rate is 4.43 events for 414 days.

The zenith angle distributions from the QE reaction are shown for $\mu$ (with and without oscillation) in Fig. \&(a) and for $\tau$ (from oscillation) in Fig. 1 (b). As expected, all of the $\tau$ produced are up-going.

We do not discuss the details of DIS production of $\tau$ because, as will be discussed later, such events are hard to identify. Nonetheless, we note that the event rate for DIS production is comparable to that of QE production. Therefore the current SuperKamiokande data set must contain about $10 \tau$ events for the oscillation parameters (9). 

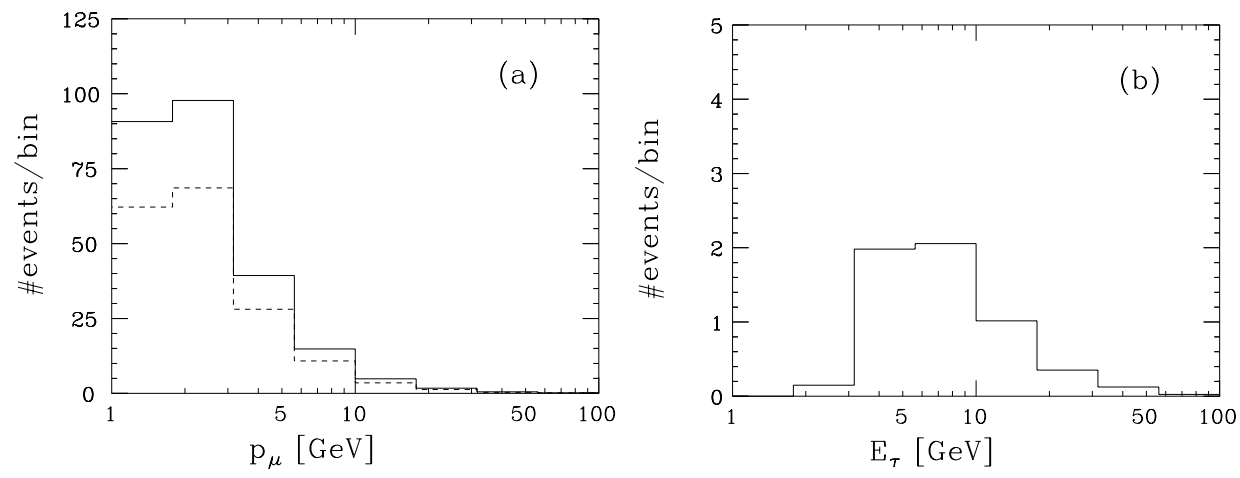

Figure 3: (a) $\mu$ and (b) $\tau$ production rates from QE reactions at SuperKamiokande for 414 days for lepton momentum greater than $1.33 \mathrm{GeV}$ (Multi-GeV). The dashed histogram in (a) and the solid histogram in (b) are the case with neutrino oscillation, with parameters given in Eq. (9).
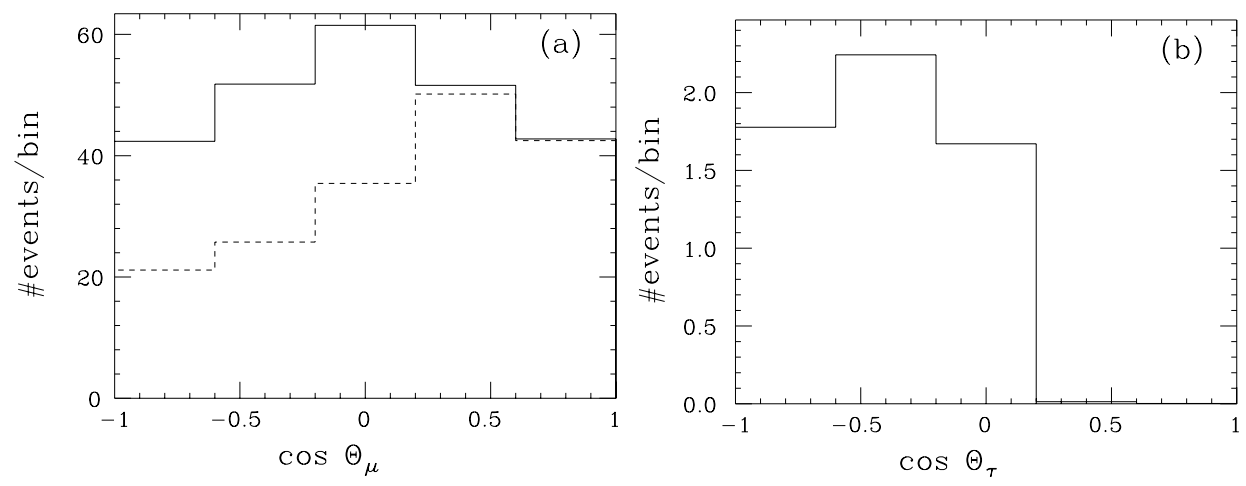

Figure 4: The zenith angle distributions in (a) $\mu$ and (b) $\tau$ production from QE reactions at Super-Kamiokande for 414 days in the multi-GeV region. The dashed histogram in (a) and the solid histogram in (b) are the case with neutrino oscillation, with parameters given in Eq. (9). 


\section{Backgrounds}

The DIS production of $\tau$ leads to multi-ring events. However, the characteristics of these events make it difficult to identify the $\tau$. The main difference from DIS CC production of $e$ and $\mu$ is the absence of an energetic charged lepton, which is difficult to establish in multi-ring events, especially for $e$. The DIS NC events do not have charged leptons and are even more similar to DIS $\tau$ events. Furthermore, the DIS cross sections for $\tau$ production in the relevant range are smaller than those for the corresponding $\mu$ production by an order of magnitude. It appears to be difficult to separate the DIS $\tau$ events from the DIS $\mu$ events, and we do not consider DIS $\tau$ production in this letter.

Instead, we focus on the QE production of $\tau$. Apart from the possible ring of the recoil nucleon, the entire event is caused by the decay products of the $\tau$. The purely leptonic decay modes, $\tau \rightarrow \nu_{\tau} \nu_{l} l$ for $l=e, \mu$, have branching fractions of $17.8 \%$, and $17.4 \%$, respectively. These events would appear the same as the QE production of $e$ or $\mu$, and hence cannot be used as the signal.

The single pion decay mode $\tau^{ \pm} \rightarrow \nu_{\tau} \pi^{ \pm}$has a branching fraction of $11.3 \%$. The physics background to this signal is from the single pion production from neutral current (NC) interaction of the neutrinos with nucleons,

$$
\begin{aligned}
& \stackrel{(-)}{\nu_{l}} n \rightarrow \stackrel{(-)}{\nu_{l}} p \pi^{-}, \\
& \stackrel{(-)}{\nu_{l}} p \rightarrow \stackrel{(-)}{\nu_{l}} n \pi^{+},
\end{aligned}
$$

where $\stackrel{(-)}{\nu_{l}}$ can be any of the active neutrino species. In the energy range of our interest, the NC production of a single pion is dominated by the production of baryon resonances with their subsequent decays into a nucleon and a pion. Rein and Sehgal (RS) [14] attempted the calculation of the resonance contributions, assuming a simple harmonic oscillator model of the baryon resonances to calculate the transition amplitudes. Even though the model itself is somewhat questionable, their calculations show reasonable agreement with data within about $20 \%$ for charged current (CC) single pion production [15]. Therefore, we can rely on RS calculations on neutral current single pion production cross sections at $20 \%$ level. The cross sections for processes (10,11) are almost flat above $E_{\nu} \gtrsim 3 \mathrm{GeV}$, both of them at $1.0 \mathrm{fb}$ [14. Unfortunately, this is larger than the expected size of the signal cross sections 
Table 1: The single rho production cross sections in fb using the resonance production cross sections in [14] and their subsequent decays into rho in [17].

\begin{tabular}{|c|cc|}
\hline & $E_{\nu}=2 \mathrm{GeV}$ & $E_{\nu}=20 \mathrm{GeV}$ \\
\hline$\nu p \rightarrow \nu n \rho^{+}$ & $0.11-0.13$ & $0.20-0.50$ \\
$\nu n \rightarrow \nu p \rho^{-}$ & $0.074-0.14$ & $0.26-0.57$ \\
\hline
\end{tabular}

Eqs. (7,8) once multiplied with the tau branching fraction to single pion of $11.3 \%$. This process also suffers from the "fake" background of charged current muon production where the muon is identified as a charged pion. This cross section is an order of magnitude larger, and is a continuum in muon energy with no features. Even though the SuperKamiokande Collaboration is working on the discrimination of charged pions from muons, which appears feasible based on their beam-based study at KEK [16], identification of $\tau$ in the pion mode appears quite difficult.

The $\tau \rightarrow \nu_{\tau} \rho^{-}$mode has a much larger branching fraction of $25.2 \%$. We find this mode much more promising than the pion mode. Unfortunately, the total cross sections of the physics background, the NC single-rho production, are neither measured nor calculated. We therefore extend the RS calculations to rho production by summing over the resonance production cross sections given in their TABLE IV multiplied by the branching fractions into rho from Particle Data Group [17. Our calculations neglect the interference between resonances as well as the continuum contribution, but we have verified that our naive method gives cross sections for the single pion production which agrees with their full calculations within about 10\%. The larger uncertainty, however, results from the lack of data on the rho branching fractions of baryon resonances. We therefore give conservative and optimistic estimates of the NC single-rho production cross sections by taking the upper (up to $100 \%$ if not measured) and lower (down to $0 \%$ if not measured) ends of the rho branching fractions, respectively. The results of our calculations are shown in the Table 1. The uncertainties are unfortunately rather large. It is encouraging, however, that these cross sections are up to an order of magnitude lower than the signal cross sections of QE $\tau$ production.

There are other "fake" backgrounds to the single rho production from $\tau$ appearance. The most important one is the charged current $\nu_{\mu}$ event with a single neutral pion production, i.e. $\nu_{\mu} n \rightarrow \mu^{-} p \pi^{0}$ or $\bar{\nu}_{\mu} p \rightarrow \mu^{+} n \pi^{0}$, where the muon is misidentified as a charged pion and the invariant mass of the 
charged pion and the neutral pion falls into the range of the rho mass. The relevant cross sections are

$$
\begin{aligned}
\sigma\left(\nu_{\mu} n \rightarrow \mu^{-} p \pi^{0}\right) & \simeq 3 \mathrm{fb} \\
\sigma\left(\bar{\nu}_{\mu} p \rightarrow \mu^{+} n \pi^{0}\right) & \simeq 2 \mathrm{fb} .
\end{aligned}
$$

Comparing with the signal cross sections, Eqs. (77,8), and $\tau \rightarrow \nu_{\tau} \rho$ branching fraction of $25.2 \%$, we would like to achieve a rejection factor on $\mu^{ \pm} \pi^{0}$ production by an order of magnitude (keeping $S / N \gtrsim 3$ ). Even though we do not know the realistic number for it, it is reasonable to expect that the combination of the mass cut on $\pi^{ \pm} \pi^{0}$ within the $m_{\rho}$ range together with $\pi^{ \pm} / \mu^{ \pm}$ discrimination being discussed [16], this level of rejection can be achieved.

The next class of background events to discuss is the deep inelastic events with $\rho$ production. The inclusive production rate of $\rho^{ \pm}$from neutral current reactions is not measured. However, there is a reported rate of inclusive production rate of $\rho^{0}$ from charged current reactions, which give $\rho$ multiplicity of about 0.1 [18] in the energy range of our interest. Assuming the comparable $\rho^{ \pm}$production rate from neutral current reactions, together with the total neutral current cross section of about $7 \mathrm{fb}$ at $E_{\nu} \sim 5 \mathrm{GeV}$, the inclusive $\rho$ production cross section would be about $0.7 \mathrm{fb}$. Since these events are likely to be associated with production of a few other pions, the requirement of single $\rho$ production is expected to suppress this background further. Overall, the background rate from deep inelastic $\rho$ production is much smaller than the signal.

Another possible source of background is the neutral current events of multi-pion production (DIS) where only one neutral pion is produced and only one charged pion is above the Čerenkov threshold. The estimate of this background depends sensitively to the detector capability and is beyond the scope of this paper. It should be noted, however, that there is no particular reason for the neutral and charged pions to form an invariant mass in the rho mass range for this process, and the continuum DIS process itself is relatively low at this energy range.

As we have seen above, the estimates of the background are quite crude at best, primarily because of the lack of relevant data at this energy region.

The efficiency in reconstructing rho is crucial in the study of $\tau$ appearance. The closest study we could find in the literature is the search for proton decay $p \rightarrow \rho^{+} \nu$ by Kamiokande experiment 19. The efficiency was unfortunately low: $15 \%$. We can only hope that the Super-Kamiokande can achieve higher 
efficiency thanks to much better granularity of the detector. It is encouraging to quote the high efficiency of $54 \%$ quoted by the IMB collaboration in their search for $p \rightarrow \rho^{ \pm} \nu$ [20].

Recall that we discussed only the QE contribution to $\tau$ production. There are, however, additional processes of $\tau$ production with additional particles where they are below the Cerenkov threshold. This adds more events to the single $\tau$ production. On the other hand, the same enhancement occurs also for the background. It is not clear what the exact situation is.

Let us emphasize that the background cross section can be measured by the near detector in the $\mathrm{K} 2 \mathrm{~K}$ experiment, the long-baseline experiment from KEK to Super-Kamiokande. The near detector consists of a 1 kt water Cerenkov detector followed by a tracking device, a calorimeter and a muon chamber. With the water Cerenkov detector they can study the "single$\rho "$ events. Then the uncertainty in the estimated background level will be significantly reduced.

\section{Statistical Significance of $\tau$ Appearance}

We estimate the statistical significance for a $\tau$ appearance signal at SuperKamiokande. Suppose that the actual total event rate for signal and background $\rho^{ \pm}$events is $S$ and $B$ events per year, and that a fraction $\epsilon$ of these are reconstructed. The background events will be up-down symmetric, whereas the signal events are dominantly up-going. We assume a value for $\Delta m^{2}$ such that all signal events are up-going. With $N$ years of data there will be $N \epsilon B / 2$ down-going events and $N \epsilon(B / 2+S)$ up-going events.

For quasi-elastic $\tau$ production we have calculated $S=1.0$ for $\sin ^{2} 2 \theta=1$; while for single $\rho$ production we estimate $B=0.4$, using the extension of the Rein-Sehgal resonance calculation discussed in the previous section. Hence with $\epsilon=0.5$, and $N=4(8)$ we expect 0.4 (0.8) downgoing events and 2.4 (4.8) up-going events. We would like to determine the probability that the up-going events are caused by a statistical fluctuation of the background; however, it is difficult to do this precisely because there are so few down-going events that the background is very poorly determined. Hence we will assume that the background has been determined from measurements in a neutrino beamline, such as at the near detector of the K2K experiment. In this case, for the observed excess of up-going events compared to down-going events to be due to a statistical fluctuation of the background requires a fluctuation 
of $\sqrt{2 N \epsilon} S / \sqrt{B}$ standard deviations. With $S=1$ and $B=0.4$, this gives a $4.6 \sigma$ effect for $N \epsilon=4$. As discussed earlier, there are other contributions to both $S$ and $B$, so that it is probable that the $S / \sqrt{B}$ value used here, 1.6, is not correct. For $S / \sqrt{B}=(1.0,1.6,2.5)$, the signal is a $(2.8,4.6,7.1) \sigma$ effect, for $N \epsilon=4$. Hence a significant result is only expected from a multi-year search with high $\rho$ reconstruction efficiency. The event rates are low, so that in practice the signal could be worse or better depending on whether the number of observed events happens to fluctuate above or below the mean expected number.

\section{Conclusion}

We have studied the prospect of detecting $\tau$ appearance in Super-Kamiokande detector, from the oscillation of atmospheric $\nu_{\mu}$ to $\nu_{\tau}$, and have identified $\tau \rightarrow \nu_{\tau} \rho^{ \pm}$as a promising detection mode. The event rate, however, is low: about 1.0 event per year from quasi-elastic production. There are possibly some additional contributions from non-QE processes where the other products (pions) are below the Cerenkov threshold. We estimated rates for background processes, and, even though the estimates have large uncertainties, none of the background processes appear larger than the signal. The $S / N$ can range anywhere between $\sim 1 / 3$ to 1 . This uncertainty will be reduced by direct measurements of the background at the K2K experiment. Although challenging, with 5 to 10 years of data, the detection of $\tau$ appearance from

atmospheric neutrinos may be possible, for example, with an observation of 5 upward going events and 1 downward.

\section{Acknowledgements}

HM thanks Michael S. Chanowitz, Stuart J. Freedman, Michael Jones, Takaaki Kajita, Kam-Biu Luk, Gil Shapiro, and Henry W. Sobel for useful discussions. This work was supported in part by the U.S. Department of Energy under Contracts DE-AC03-76SF00098, in part by the National Science Foundation under grant PHY-95-14797. HM was also supported by Alfred P. Sloan Foundation. 


\section{References}

[1] Super-Kamiokande Collaboration (Y. Fukuda et al.), Phys. Lett. B433, 9 (1998), hep-ex/9803006;

Super-Kamiokande Collaboration (Y. Fukuda et al.), hep-ex/9805006;

Super-Kamiokande Collaboration (Y. Fukuda et al.), Phys. Rev. Lett. 81, 1562 (1998), hep-ex/9807003;

T. Kajita, talk presented at The XVIIIth International Conference on Neutrino Physics and Astrophysics (NEUTRINO'98), Takayama, Japan, 4-9 June, 1998, hep-ex/9810001.

[2] Kamiokande-II Collaboration (K.S. Hirata et al.), Phys. Lett. 205B, 416 (1988);

Kamiokande-II Collaboration (K.S. Hirata et al.), Phys. Lett. B280, 146 (1992);

Kamiokande Collaboration (Y. Fukuda et al.), Phys. Lett. B335, 237 (1994);

Kamiokande Collaboration (S. Hatakeyama et al.), Phys. Rev. Lett. 81, 2016 (1998), hep-ex/9806038.

[3] IMB Collaboration (D. Casper et al.), Phys. Rev. Lett. 662561 (1991); IMB Collaboration (R. Becker-Szendy et al.), Phys. Rev. D46 3720 (1992).

[4] T. Kafka, talk presented at 5th International Workshop on Topics in Astroparticle and Underground Physics (TAUP 97), Gran Sasso, Italy, 7-11 Sep 1997, hep-ph/9712281;

E. Peterson, talk presented at The XVIIIth International Conference on Neutrino Physics and Astrophysics (NEUTRINO'98), Takayama, Japan, 4-9 June, 1998;

H. Gallagher, parallel session talk presented at The 29th International Conference on High-Energy Physics (ICHEP 98), 23-29 Jul 1998, Vancouver, Canada.

[5] MACRO Collaboration (M. Ambrosio et al.), Phys. Lett. B434, 451 (1998), hep-ex/9807005.

[6] V. Barger, T. J. Weiler, and K. Whisnant, hep-ph/9807319;

G.L. Fogli, E. Lisi, A. Marrone and G. Scioscia, hep-ph/9808205. 
[7] CHOOZ Collaboration (M. Apollonio et al.), Phys. Lett. B420 397 (1998).

[8] C.H. Llewellyn Smith, Phys. Rept. 3, 261 (1972).

[9] T. Kitagaki et al., Phys. Rev. D28, 436 (1983).

[10] A.D. Martin, W.J. Stirling, and R.G. Roberts, Phys. Rev. D51, 4756 (1995).

[11] C.H. Albright and J. Jarlskog, Nucl. Phys. B84, 467 (1975).

[12] M. Honda, T. Kajita, K. Kasahara, and S. Midorikawa, Phys. Rev. D52, 4985 (1995).

[13] E. Kearns, talk presented at Institute for Theoretical Physics Conference on Solar Neutrinos: News About SNUs, December 2-6, 1997, Institute for Theoretical Physics, University of California, Santa Barbara.

[14] D. Rein and L.M. Sehgal, Annals of Physics, 133, 79 (1981).

[15] SKAT Collaboration (H.J. Grabosch et al.), Z. Phys. C41, 527 (1989).

[16] Henry W. Sobel, private communications.

[17] Particle Data Group (C. Caso et al)., Eur.Phys.J. C3, 1 (1998).

[18] SKAT Collaboration (V.V. Ammosov et al.), Sov. J. Nucl. Phys. 46, 80 (1987).

[19] Kamiokande-II Collaboration (K.S. Hirata et al.), Phys. Lett. B220, 308 (1989).

[20] Larry Sulak, "Mini-reivew of $p$-decay: IMB, Soudan-2, other modes at Super-K," parallel session talk presented at XXIX International Conference on High Energy Physics (ICHEP), Univ. of British Columbia, Vancouver, B.C., Canada, July 23-29, 1998. 